\title{
PSICOTERAPIAS DA DEPRESSÃO NA INFÂNCIA E NA ADOLESCÊNCIA
}

\author{
PSYCHOTHERAPY IN CHILD AND \\ ADOLESCENT DEPRESSION
}

Saint Clair BAHLS ${ }^{1}$

Flávia Rocha Campos BAHLS ${ }^{2}$

\begin{abstract}
RESUMO
A depressão é uma doença comum, freqüentemente crônica, recorrente e familiar, cujo início ocorre, muitas vezes, na infância e na adolescência. E a depressão em crianças e adolescentes aparenta estar aumentando e iniciando cada vez mais precocemente, constituindo atualmente uma das principais preocupações de saúde pública. Pela razão de constituir doença grave e que normalmente prediz sua continuação ou recorrência na idade adulta, sua abordagem terapêutica é de extrema importância. $O$ atendimento adequado da depressão em crianças e adolescentes é realizado com uma aproximação multidisciplinar, envolvendo basicamente recursos psicossociais. Neste artigo revisaremos a condição atual das abordagens psicoterapêuticas empregadas no tratamento da depressão na infância e na adolescência.
\end{abstract}

Palavras-chave: depressão; infância e adolescência; tratamento psicoterápico.

\begin{abstract}
Depression is a common, chronic, recurrent, and often familial illness that frequently first occurs in childhood and adolescence. Child and adolescent depression seems to be occurring more frequently and earlier than before, and it is, nowadays an important public health problem. Because depression in youth is a serious disorder and often predicts subsequent depression in adulthood, treatment for these population is relevant. Therapeutic modes
\end{abstract}

(1) Médico Psiquiatra - Mestre em Psicologia da Infância e Adolescência e Prof. Departamento de Psicologia da UFPR e da Univ. Tuiuti do PR.

Endereço para correspondência: Saint Clair Bahls - R. Carneiro Lobo 570 conj. 1403 - Fone/fax:41-242-6132 - Curitiba, Paraná - Cep: 80240-240 - E-mail: scbahls@uol.com.br

(2) Psicóloga Clínica - Mestre em Psicologia da Infância e Adolescência 
that have been shown to be useful for depressed children and adolescents are multi-modal and include psychosocial approaches. This paper will review the present status of psychotherapy treatment of depression in children and adolescents.

Key words: depression; childhood and adolescence; psychotherapy treatment.

\section{INTRODUÇÃO}

A depressão maior em crianças e adolescentes, embora de reconhecimento recente, vem alcançando níveis epidemiológicos preocupantes, com taxas de prevalência-ano em crianças entre 0,4 a 3,0\%, e nos adolescentes de 3,3 a $12,4 \%$, sendo atualmente considerada um problema de saúde pública. Também se observa um aumento crescente da prevalênciae um início cada vez mais precoce (Bahls, 2002a). Costuma causar prejuízo e limitações e traz um alto risco de suicídio, especialmente entre os adolescentes, existindo a clara necessidade de abordagens terapêuticas eficazes para seu manejo.

No tratamento da depressão em crianças e adolescentes, além do paciente, deve fazer parte do plano de tratamento a família e o ambiente, especialmente o escolar, incluindo aspectos multidisciplinares e multimodais de abordagem; e apresenta três objetivos principais: remissão dos sintomas, restabelecer o pleno funcionamento e prevenir o reaparecimento do quadro clínico (Emslie \& Mayes, 1999; Son \& Kirchner, 2000; Bahls, no prelo). As principais abordagens das depressões infanto-juvenis se dividem em psicofarmacológicas e psicossociais; e entre as psicossociais predominam as intervenções psicoterapêuticas.

As psicoterapias são amplamente indicadas na depressão infanto-juvenil, tanto nos casos de intensidade sintomatológica leve a moderada, como associada à psicofarmacologia nos casos mais graves (Son \& Kirchner, 2000; Birmaher et al, 1998). As mais utilizadas são a cognitivo-comportamental, a de orientação psicodinâmica e a interpessoal. Algumas em formato individual, grupal e familiar. Também as orientações psicoeducacionais são, atualmente, bastante empregadas e úteis (Sadler, 1991; Myiazaki \& Silvares, 1997; Schestatsky \& Fleck, 1999; Ito \& Lotufo Neto, 2000). Entretanto, poucos são os ensaios clínicos controlados existentes.

Neste artigo faremos uma revisão destas formas terapêuticas no tratamento da depressão na infância e na adolescência. Será citada brevemente a terapia cognitivo-comportamental, pois, já foi o assunto de recente publicação de um dos autores (Bahls, no prelo). Foi empregado o sistema Medline, no período de 1966 a 2002, com as palavras-chave: psicoterapia, crianças, adolescentes e depressão; e também foi utilizada a busca manual de literatura.

\section{TERAPIA COGNITIVO-COMPORTAMENTAL (TCC)}

A terapia cognitivo-comportamental resulta da associação das duas estratégias em seu emprego clínico, baseado na compreensão de que cognições mais saudáveis conduzem a padrões de comportamento mais adaptados e vice-versa (Kazdin \& Marciano, 1998; Schestatsky \& Fleck, 1999; Bahls, no prelo). É a forma terapêutica mais testada no tratamento da depressão infanto-juvenil, com ensaios clínicos controlados e randomizados (ECR) desde 1980 com o estudo pioneiro de Butler e colaboradores.

Reunimos em revisão sistematizada recente (Bahls, no prelo) quatorze ensaios clínicos controlados e randomizados com TCC em crianças e adolescentes deprimidos. $\mathrm{Na}$ 
fase aguda do tratamento, a TCC foi superior aos grupos controles e semelhante às outras modalidades terapêuticas comparadas. E nos poucos estudos de seguimento mostrou-se tão eficaz em prevenir o reaparecimento do quadro depressivo quanto as outras terapias usadas como controle.

\section{TERAPIA INTERPESSOAL (TIP)}

A terapia interpessoal baseia-se nas contribuições de Adolf Meyer, Harry Stack Sullivan e John Bowlby, e foi sistematizada por Mirna Weissman e Gerald Klerman e colaboradores. Apóia-se nas observações de que relacionamentos interpessoais saudáveis participam na prevenção da depressão e muitas vezes a ruptura dos mesmos representa uma função importante no desenvolvimento da depressão (Weissman, 1997; Ito \& Lotufo Neto, 2000; Curry, 2001). Entretanto a TIP não considera os fatores interpessoais como a causa da depressão, mas entende que seu início, a resposta ao tratamento e o prognóstico são influenciados pelas relações do deprimido com suas pessoas significativas (Rossello \& Bernal, 1999: Schestatsky \& Fleck, 1999).

É uma forma de terapia que foca sua intervenção em situações de conflitos e problemas atuais do paciente. Procura ajudar os pacientes a entender seus problemas presentes dentro do contexto de suas relações interpessoais, ensinando o monitoramento dos sintomas depressivos, questionamento exploratório, esclarecimento de problemas, habilidades de comunicação e identificação da relação entre afetos e acontecimentos (Weissman, 1997; Kazdin \& Marciano, 1998; Brent \& Birmaher, 2002).

Tem estrutura breve e focal, estando indicada para pacientes com depressão aguda não psicótica ou bipolar e que não necessitem de internamento. As dificuldades abordadas são divididas em quatro categorias, geralmente chamadas de "áreas-problema": luto, conflitos interpessoais, mudanças de papel (transição) e déficits interpessoais. Tem duração preestabelecida de aproximadamente 16 sessões em quatro meses e deve concentrar-se em uma ou duas áreas problemáticas. Possui três fases: a) inicial: com finalidade de fazer a anmenese, explicar a doença e o processo terapêutico, fazer um inventário interpessoal, estabelecer um foco e o plano de tratamento; b) intermediária: consiste no desenvolvimento do processo terapêutico com a investigação das áreas-problema definidas na fase inicial, na abordagem dos problemas interpessoais selecionados e seu monitoramento e c) final: em que se consolidam os avanços conquistados com o desenvolvimento da capacidade de identificar e lidar com novos sintomas depressivos e se discute o final da terapia.

Ainda pode ser utilizada na forma de tratamento de manutenção após o tratamento da fase aguda, com a finalidade de manter a recuperação e reduzir a vulnerabilidade a futuros episódios. Procura reforçar o estado de saúde do contexto psicossocial alcançado pelo paciente, geralmente com uma freqüência mensal de atendimento (Moreau et al, 1991; Weissman, 1997; Ito \& Lotufo Neto, 2000). Também pode associar-se com a compreensão psicodinâmica, com técnicas cognitivo-comportamentais, com medicamentos e envolver membros da família, quando necessário (Rossello \& Bernal, 1999; Schestatsky \& Fleck, 1999).

A TIP foi revisada por Moreau et al. em 1991 para ser utilizada em adolescentes abrangendo questões normalmente enfrentadas por eles, tais como: afastamento dos pais, problemas com autoridade, desenvolvimento e manutenção de relações duais, perdas ou morte de parentes e/ou amigos, pressão de grupo de pares e situações difíceis relacionadas com as famílias de pai único. Mufson et al. (1994) demonstraram em um estudo aberto com quatorze adolescentes deprimidas, todas do sexo feminino, que todas não mais preencheram critérios para a patologia ao final de doze semanas de tratamento, melhorando significativamente 
também o funcionamento social e global. Em estudo naturalístico com um ano de seguimento, Mufson e Fairbanks (1996), acompanhando 10 adolescentes do estudo anterior, constataram que somente uma apresentou reaparecimento do quadro clínico. Novamente o grupo da Universidade de Columbia (EUA), com Mufson et al. em 1999, acompanhou em estudo clínico controlado e randomizado 48 adolescentes (12 a 18 anos) com diagnóstico de depressão maior, atendidos semanalmente durante doze semanas com TIP ou acompanhamento clínico regular de apoio (grupo controle). Trinta e dois participantes concluíram o estudo e $75 \%$ no grupo TIP versus $46 \%$ no grupo controle se recuperaram ao final do estudo, com notável melhora na sintomatologia depressiva, no funcionamento geral e na solução de problemas nos participantes da TIP. Concluíram que a TIP é uma forma de terapia em adolescentes deprimidos de boa aceitabilidade, simples e eficaz. O outro estudo clínico randomizado foi realizado em Porto Rico por Rossello e Bernal (1999) comparando TIP, TCC e lista de espera em 71 adolescentes deprimidos (13 a 17 anos), no período de 12 semanas, com resultados de melhora em $82 \%$ do grupo TIP e de $59 \%$ no grupo TCC, resultados superiores ao grupo controle e sem diferença estatisticamente significativa entre eles. Num período de três meses, não houve diferença entre os dois grupos que receberam tratamento ativo.

Portanto, tem sido sugerida sua eficácia no tratamento agudo de adolescentes deprimidos e aparenta prevenir o reaparecimento do quadro clínico (Weissman, 1997; Birmaher et al, 1998; Curry, 2001; Brent \& Birmaher, 2002). Parece promissora, apesar de existir somente dois ensaios clínicos controlados até o momento e ainda não ter sido estudada em crianças deprimidas.

\section{TERAPIAS DE ORIENTAÇÃO PSICODINÂMICA (TOP)}

Abrange todas as formas de terapias baseadas nos princípios psicanalíticos. Investiga as fantasias e desejos reprimidos, estabelecidos no inconsciente, como determinantes dos conflitos intrapsíquicos. E o ego lida com estes conflitos (a disputa entre impulsos sexuais e agressivos com o superego e a realidade) utilizando os chamados mecanismos de defesa (Freud, 1936). Na eventualidade de os recursos egóicos falharem no manejo adequado destes conflitos, ocorrerá o aparecimento de sintomas e quadros clínicos psicopatológicos.

No processo terapêutico, o paciente é orientado a expressar seus pensamentos e sentimentos abertamente e de maneira não dirigida, com a finalidade de reexperimentar traumas precoces na relação analítica. Tem o terapeuta a função de esclarecer, trazendo compreensão consciente aos conflitos, por meio do manejo transferencial. A transferência permite ao analista uma oportunidade inestimável de explorar o passado e o inconsciente do paciente, assim como a contratransferência oferece corretas orientações para a compreensão do processo psicanalítico (Freud,,1912; Moore \& Fine, 1968). Desde os estudos de M. Klein, aceita-se que a transferência ocorre na terapia com crianças e adolescentes, mesmo havendo a participação ampla e ativa da figura dos pais na vida dos mesmos (Chethik, 1989).

As TOP podem assumir a forma de atendimento breve e costumam compartilhar as seguintes características: foco centrado em problemas de perdas e separações, seleção de pacientes motivados e ênfase na elaboração da raiva e pesar pela finalização sempre iminente da terapia. Devem manter a atenção clínica no conflito dinâmico primário e promover o desenvolvimento do ego por meio da interpretação (Austin \& Inderbitzin,1983; Schestatsky \& Fleck, 1999).

Em crianças de até dois anos de idade, a depressão freqüentemente representa uma disfunção familiar e/ou uma psicopatologia nos pais. Nesta faixa etária, habitualmente a terapia deve ser realizada com o grupo pais-criança, e o terapeuta desempenha um papel mais ativo no processo de tratamento. Nas crianças entre três 
a seis anos (pré-escolares), nas quais a linguagem preferencial é o brincar, esta costuma ser a ferramenta psicoterapêutica empregada e os pais também devem ser atendidos e orientados periodicamente (Shaffi \& Shaffi, 1992).

Crianças deprimidas na faixa escolar (7 a 12 anos) costumam deslocar para a terapia seus sentimentos patológicos e sintomas de raiva, desesperança e isolamento (Bahls, 2002b), tornando a tarefa terapêutica especialmente árdua. Um recurso usado no início da terapia, em que é habitual a presença de maior resistência pelo paciente e até mesmo atitudes desafiadoras podem ocorrer, são jogos estruturados e não ameaçadores, com a finalidade de facilitar a relação terapeuta-paciente. É importante destacar que neste período as crianças ainda se encontram no estágio pré-operacional da inteligência, que funciona, muitas vezes, de forma concreta e, como o pensamento abstrato usualmente começa a predominar por volta dos doze anos de idade, o terapeuta deve focar seu trabalho no aqui e agora, procurando tornar o paciente mais flexível em relação a sua aderência a mecanismos defensivos patológicos. Visitas ao lar e à escola podem ser bastante úteis, assim como um trabalho de orientação aos pais e mestres. Por exemplo, quando a criança deprimida começa a verbalizar ou apresentar atitudes agressivas, geralmente indicando o começo de sua recuperação, é necessário, neste momento, a compreensão dos pais e professores para o bom desfecho terapêutico (Shaffi \& Shaffi, 1992; Gabbard, 2001).

Adolescentes deprimidos costumam ser um desafio para a terapia devido à instabilidade afetiva, ao narcisismo, à descrença nos adultos, ao isolamento, ao conflito dependência versus independência e à aguda sensibilidade à opinião e aceitação de seus pares. O terapeuta deve procurar não se superidentificar ou manter um distanciamento excessivo em relação ao adolescente, sendo franco e direto, pois o aparecimento do pensamento abstrato e da intelectualização permite o uso da terapia pela palavra. No entanto, devido a possíveis regressões próprias do quadro depressivo, algumas vezes o adolescente pode apresentar dificuldades cognitivas e comportamentais, às quais o terapeuta deve estar atento, observando também atitudes não verbais. Tal como as crianças os pais devem ser avaliados e corretamente orientados (Shaffi \& Shaffi, 1992). A ideação e o comportamento suicida devem despertar especial consideração e cuidado na terapia de adolescentes deprimidos (Gabbard, 2001; Bahls \& Bahls, 2002).

Em estudo aberto inglês, Baruch et al. (1998), com 130 jovens (12 a 24 anos) que buscaram atendimento em um centro comunitário de psicoterapia psicanalítica, observaram como fatores previsíveis que levam ao abandono da terapia: menor idade e maior grau de problemas externos (agressão e delinqüência), problemas escolares e um grau moderado a grave de problemas de conduta e hiperatividade. Este estudo incluiu adultos jovens e não incluiu somente quadros depressivos.

As TOP são indicadas nos casos de jovens interessados e preocupados com seus conflitos e questões psicológicas e são úteis na compreensão de sentimentos e impulsos, no aumento da auto-estima, na mudança de padrões mal-adaptativos de comportamento, na interação de maneira mais saudável com as pessoas e para lidar com conflitos passados e atuais (Scivoletto et al, 1994; Birmaher et al, 1998; Gabbard, 2001). Até o momento, desconhecemos a existência de estudos clínicos controlados com as TOP em depressão de crianças e adolescentes não havendo, portanto, nenhuma evidência científica que as sustente. Podem ser adequadas em alguns casos, sendo que a recomendação clínica tem sido pela sua forma breve de intervenção.

\section{PSICOTERAPIAS DE GRUPO}

Atribui-se a Slavson, em 1934, o início das terapias de grupo com crianças (Rachman \& Raubolt, 1984). Posteriormente, emergiram várias 
formas de terapia de grupo para crianças e adolescentes; entretanto, ainda é subutilizada, principalmente na modalidade ambulatorial.

Crianças e adolescentes deprimidos tendem a ter interações prejudicadas, as crianças sendo freqüentemente denominadas de choronas ("manteigas derretidas") e os adolescentes de problemáticos ("aborrecentes"), o que os leva a diminuir mais ainda a auto-estima e provoca rejeição e críticas, aumentando a sensação de inadequação e piorando o quadro clínico. A terapia de grupo oferece uma oportunidade de explorar a dimensão social da doença e desenvolver atitudes, modos de interação e habilidades mais funcionais e gratificantes, sendo particularmente útil em promover habilidades sociais e fortalecer a auto-imagem (Baxter \& Kennedy, 1992; Beeferman \& Orvaschel, 1994). O conhecimento de que outros também compartilham problemas semelhantes foi denominado por Yalom (1975) de "senso de universalidade", e representa um importamte recurso dos grupos de tratamento. Traz suporte e reasseguramento aos jovens com depressão, muitas vezes, permitindo a exploração segura de seus sentimentos e experiências no ambiente terapêutico. Crianças escolares costumam receber jogos e brincadeiras como as principais ferramentas terapêuticas em seus grupos e, à medida que a idade avança, vai sendo possível o uso de manejos verbais.

Adolescentes deprimidos apresentam uma especial dificuldade em expressar seus sentimentos e experiências interiores e as interações disponíveis no grupo de terapia podem facilitar esta expressão, oferecendo oportuni-dades de avaliar suas autopercepções com a observação dos seus pares sobre eles. Dentro de um ambiente protegido, favorecido pelo grupo terapêutico, podem desenvolver uma consciência crescente de seu comportamento e do impacto causado nos outros. A psicoterapia de grupo costuma ser útil em adolescentes, pois estes podem aceitar melhor confrontações e intervenções vindas de colegas do que do terapeuta em atendimento individual. Os grupos oferecem maior testagem da realidade e o terapeuta pode observar diretamente a interação entre o adolescente e seus pares (Baxter \& Kennedy, 1992; Scivoletto et al, 1994). Entretanto, é comum o terapeuta deparar-se constantemente com atitudes desafiadoras que necessitam de um manejo firme relacionado aos limites e regras do grupo.

Alguns profissionais têm como rotina encaminhar seus pacientes deprimidos para tratamento grupal só após um período inicial de acompanhamento individual, quando já estejam relativamente aliviados dos sintomas de autodepreciação, hipersensibilidade às opiniões de outros, sentimento de rejeição e dificuldades de socialização.

Baxter e Kennedy (1992) destacam três fases do tratamento grupal para jovens deprimidos: a) fase inicial - o paciente fica mais observando que participando, aprende as normas e expectativas do grupo e procura reconhecer o nível de confiabilidade do grupo; b) fase de compromisso e investimento - inicia quando o jovem começa a sentir-se parte integrante do grupo e identifica-se com os objetivos terapêuticos, estabelecendo a chamada "aliança terapêutica", em que se desenvolverá a principal tarefa do processo de tratamento; e c) fase de separação e conclusão - ocorre após um período de mudanças consistentes por parte do paciente e baseia-se em favorecer o processo final de consolidação de habilidades conquistadas, permitindo o afastamento de forma saudável do grupo terapêutico.

Jovens depressivos vêm sendo crescentemente atendidos em grupo, tanto de forma primária como paralelamente à terapia individual ou psicofarmacoterapia. Alguns profissionais recomendam formar grupos homogêneos, considerando patologia, gênero e idades semelhantes, porém, não existe consenso neste item das terapias infanto-juvenis de grupo e várias modalidades (orientações teóricas diversas) podem ser utilizadas. Nos estudos clínicos controlados nesta população, com as técnicas cognitivo-comportamentais e interpes- 
soais, o principal formato empregado foi o grupal (Bahls, no prelo).

\section{TERAPIA FAMILIAR E PSICOEDUCAÇÃO}

A terapia familiar objetiva atender situações clínicas em que ocorrem interações inadequadas entre pais-filhos como questões centrais no desenvolvimento e/ou manutenção da sintomatologia depressiva (Kazdin \& Marciano, 1998). Direciona-se, preferentemente, à modificação de padrões mal-adaptados de comportamento e comunicação verbal dentro da família. Pode ser utilizada em associação com outras abordagens, especialmente em situações de conflito familiar importante (Scivoletto et al, 1994). Baseado no já bem estabelecido conhecimento do padrão familiar na depressão, o diagnóstico em criança e adolescente deve necessariamente buscar a identificação de familiares que também possam apresentar a patologia, fazendo a abordagem e o tratamento da família por inteiro (Son \& Kirchner, 2000; Curry, 2001). Em seu estudo com jovens depressivos, Hammen et al. (1999) concluíram que o ambiente familiar muitas vezes é altamente disfuncional e Birmaher et al. (2000), em estudo de acompanhamento de dois anos com adolescentes deprimidos, sugerem que a diminuição de conflitos familiares é um componente importante na recuperação e na prevenção de recorrências. O primeiro estudo controlado de tratamento de adolescentes deprimidos que incluiu uma abordagem familiar foi o de Lewinsohn et al. (1990), com TCC, e não encontrou diferença significativa nos resultados entre o grupo pais-adolescentes e o grupo só de adolescentes. Este estudo foi posteriormente replicado por Clarke et al. (1999), quando novamente não ocorreu diferença estatisticamente significativa entre a abordagem somente com adolescentes e a que incluiu os pais. E, no estudo de Brent et al. (1997), os resultados do tratamento na fase aguda foram superiores com TCC quando comparados com terapia familiar sistêmica-comportamental. E entretanto, ao final da avaliação de dois anos de seguimento, os resultados foram equivalentes. Embora os poucos estudos controlados com abordagem familiar não tenham demonstrado superioridade em relação às abordagens somente dos jovens com depressão, esta é seguramente uma área terapêutica promissora e que deve receber especial atenção nas próximas pesquisas.

A compreensão, por parte do paciente e seus familiares, da patologia, seus sintomas, evolução e tratamento costuma melhorar a adesão ao tratamento e os índices de recuperação. $\mathrm{Na}$ abordagem psicoeducacional objetiva-se, portanto, o esclarecimento e a compreensão da patologia, orientando sobre sua dimensão psicobiológica e também melhorando a adesão ao tratamento (Son \& Kirchner, 2000). Coordenadores do Oregon Adolescent Depression Project, Brent e Birmaher (2002) compreendem a psicoeducação no tratamento de crianças e adolescentes deprimidos como um elemento essencial no arsenal terapêutico. Diminuindo a vergonha, a tendência de acusações e "busca de culpados" e corrigindo idéias equivocadas sobre a origem da doença, oferece a possibilidade de substituir atitudes punitivas por asseguramento e suporte adequados.

Além das informações oferecidas, a psicoeducação pretende transformar os familiares e amigos em colaboradores ativos do tratamento com a compreensão da depressão como uma doença, reconhecendo e identificando o afeto alterado, encaminhando déficits psicossociais e aprendendo a importância da adesão ao tratamento. A família deve ser estimulada a participar na elaboração do plano de tratamento, ajudando a definir áreas e comportamentos-alvo de mudanças. Por meio dessa abordagem, é possivel que os pais possam identificar seus próprios sintomas depressivos, pois as pesquisas têm demonstrado que, na época do tratamento da depressão de um adolescente, entre $30 \%$ a $50 \%$ possuem um dos pais também padecendo de doença depressiva (Birmaher et al, 1998; Emslie \& Mayes, 1999). 


\section{CONCLUSÕES}

Atualmente, é conhecida, a relevância da depressão em crianças e especialmente nos adolescentes. Todavia, a compreensão dos recursos psicoterapêuticos destinados a esta população ainda se encontra em fase incipiente, oferecendo mais indagações do que respostas. Muito poucos são os estudos clínicos controlados, ainda sujeitos a imprecisões e exigindo cautela na interpretação de seus resultados, mas observa-se que este panorama, felizmente, está mudando. Sem dúvida, o emprego de pesquisas controladas e randomizadas não é tarefa simples na área das psicoterapias e nem todas as modalidades conseguem preencher os critérios exigidos para tais estudos. Segundo a Task Force on Promotion and Dissemination of Psychological Procedures (1995), os aspectos a serem preenchidos pelos estudos em psicoterapias são: a) um modelo teórico coerente; b) um manual de tratamento com métodos específicos de procedimento; c) um formato de tempo limitado e d) medidas específicas de avaliação de resultados. E as psicoterapias que desejem integrar modelos científicos de tratamento obrigatoriamente necessitarão adequar-se a estes requisitos.

Algumas limitações observadas nesta revisão se devem ao fato de que a depressão requer tratamento em todas as suas fases evolutivas, e as pesquisas com jovens deprimidos oferecem poucos resultados e, na grande maioria, referentes somente à fase aguda da patologia. Existem raros estudos de avaliação de tratamento nas fases de continuação e manutenção. A maioria das intervenções utilizadas deriva de técnicas empregadas em populações adultas, 0 que deixa clara a falta de compreensão desenvolvimental que deveriaidentificar condições próprias de cada etapa do crescimento emocional. Esta perspectiva desenvolvimental pode requerer desenhos de intervenção e pesquisa específicos em diferentes momentos na infância e na adolescência. E ainda existe muito pouco conhecimento advindo das pesquisas sobre a importância do envolvimento dos pais e da famíla nos processos terapêuticos.

Também se observa que os critérios de exclusão comumente empregados nas pesquisas acabam por definir amostras não clinicamente significativas, por exemplo, a exclusão de comorbidades que podem ocorrer em até $80 \%$ de crianças e adolescentes deprimidos (Bahls, 2002b). Praticamente não existem estudos comparativos de eficácia entre as formas de psicoterapia empregadas no tratamento desta população, e os dados atuais, retirados dos poucos estudos realizados, sugerem uma eficácia equivalente entre elas. Há muito poucos estudos com a população infantil e raros são os pesquisadores que se interessam pelo aspecto preventivo da depressão infanto-juvenil.

Em suma, as evidências atuais suportam o emprego da terapia cognitivo-comportamental (com suas várias modalidades) e da terapia interpessoal na fase aguda da depressão infanto-juvenil. A melhora clínica na população adolescente com estas intervenções apresentou resultados entre $50 \%$ a $65 \%$ (Bahls, no prelo; Michael \& Crowley, 2001). Todo o restante são somente indagações, portanto, são necessárias muito mais pesquisas relativas ao tratamento de crianças e adolescentes deprimidos.

\section{REFERÊNCIAS BIBLIOGRÁFICAS}

AUSTIN, L. \& Inderbitzin, L.B. (1983). Brief psychotherapy in late adolescence: a psychodynamic and developmental approach. American Journal of Psychotherapy 37 (2): 202-209.

BAHLS, S.C. (2002a). Epidemiology of depressive symptoms in adolescents of a public school in Curitiba, Brazil. Revista Brasileira de Psiquiatria, 24 (2): 63-67.

BAHLS, S.C. (2002b). Aspectos clínicos da depressão na infância e adolescência. Jornal de Pediatria 78 (5): 359-366.

BAHLS, S.C. (no prelo). Uma revisão sobre a terapia cognitivo-comportamental da 
depressão na infância e na adolescência. Psicologia Argumento. Curitiba, PR.

BAHLS, S.C. \& Bahls F.R.C. (2002). Depressão na adolescência: características clínicas. Interação 6: 49-57.

BARUCH, G;, Gerber, A. \& Fearon, P. (1998). Adolescents who drop out of psychotherapy at a community-based psychotherapy centre: a preliminary investigation of the characteristics of early drop-outs, late drop-outs and those who continue treatment. British Journal of Medical Psychology 71 (3): 233-245.

BAXTER, R.F. \& Kennedy, J.F. (1992). Group Therapy of Depression. In: M. Shafii, S. L. Shafii (eds.) Clinical Guide to Depression in Children and Adolescents. Washington: American Psychiatric Press. pp. 177-195.

BEEFERMAN, D. \& Orvaschel, H. (1994). Group psychotherapy for depressed adolescent: a critical review. International Journal of Group Psychotherapy 44 (4): 463-475.

BIRMAHER, B.; Brent, D.A. \& Benson, R.S. (1998). Summary of the practice parameters for the assessment and treatment of children and adolescents with depressive disorders. American Academy of Child and Adolescent Psychiatry. Journal of American Academy of Child and Adolescent Psychiatry, 37: 1234-1238.

BIRMAHER, B.; Brent, D.A., Kolko, D., Baugher, M., Bridge, J., Holder, D., lyengar, S. \& Ulloa, R.E. (2000). Clinical outcome after short-term psychotherapy for adolescents with major depressive disorder. Archives of General Psychiatry 57 (1) 29-36.

BRENT, D.A.; Holder, D.; Kolko, D.; Birmaher, B., Baugher, M., Roth, C., lyengar, S. \& Johnson, B.A. (1997). A clinical psychotherapy trial for adolescent depression comparing cognitive, family, and supportive therapy. Archives General Psychiatry, 54: 877-885.

BRENT, D.A. \& Birmaher, B. (2002). Adolescent depression. New England Journal of Medicine 347 (9): 667-671.
BUTLER, L.; Miezitis, S.; Friedman, R. \& Cole, E. (1980). The effect of two school-based intervention programs on depressive symptoms in preadolescents. American Educational Research Journal, 17: 111-119.

CHETHIK, M. (1989). Techniques of Child Therapy: Psychodynamic Strategies. New York. Guilford Press.

CLARKE, G.N.; Rohde, P.; Lewinsohn, P.M., Hops, H. \& Seeley, J.R. (1999). Cognitivebehavioral treatment of adolescent depression: efficacy of acute group treatment and booster sessions. Journal of American Academy of Child and Adolescent Psychiatry, 38 (3): 272-279.

CURRY, J.F. (2001). Specific psychotherapies for childhood and adolescent depression. Biological Psychiatry 49 (12): 1091-1100.

EMSLIE, G.J. \& Mayes, T.L. (1999). Depression in children and adolescents. A guide to diagnosis and treatment. CNS Drugs, 11 (3): 181-189.

FREUD, A. (1936). The Ego and the Mechanisms of Defense. New York: International University Press.

FREUD, S. (1912). The dynamics of transference. Standard Edition of the Complete Psychological Works of Sigmund Freud, v. 12. London: Hogarth Press.

GABBARD, G.O. (2001). Psychodynamic Psychotherapies. In G.O. Gabbard (ed.) Treatments of Psychiatric Disorders 3th. Ed. v. 2. Washington: American Psychiatric Press. pp. 1227-1245.

HAMMEN, C.; Rudolph, K.; Weisz, J.; Rao, U. \& Burge, D. (1999). The context of depression in clinical-referred youth: neglected areas in treatment. Journal of American Academy of Child and Adolescent Psychiatry, 38 (1): 64-71.

ITO, L.M. \& Lotufo Neto, F. (2000). Terapias Cognitivo-Comportamentais, Interpessoal e Construtivista. Em B. Lafer, O. P. Almeida, R. Fráguas Jr \& E. C. Miguel (eds.) Depressão 
no Ciclo da Vida. Porto Alegre: Artes Médicas. pp. 82-91.

KAZDIN, A.E. \& Marciano, P.L. (1998). Childhood and adolescent depression. In: E. Mash, R. Barkley (eds.) Treatment of Childhood Disorders, 2. ed. New York: The Guilford Press. pp. 211-248.

LEWINSOHN, P.M.; Clarke, G.N.; Hops, H. \& Andrews, J. (1990). Cognitive-behavioral treatment of depressed adolescents. Behavior Therapy, 2: 385-401.

MICHAEL, K.D. \& Crowley, S.L. (2002). How effective are treatments for child and adolescent depression? A meta-analytic review. Clinical Psychology Review 22 (2): 247-269.

MIYAZAKI, M.C.O.S. \& Silvares, E.F.M. (1997). Diagnóstico e intervenção clínica comportamental infantil: uma breve revisão. Estudos de Psicologia, 14 (1): 15-28.

MOORE, B.E. \& Fine, B.D. (1968). A Glossary of Psychoanalytic Terms and Concepts, $2^{\text {nd }}$ ed. New York: American Psychoanalytic Association.

MOREAU, D.; Mufson L.; Weissman, M.M. \& Klerman, G.L. (1991). Interpersonal psychotherapy for adolescent depression: Description of modification and preliminary application. Journal of American Academy of Child and Adolescent Psychiatry, 30: 642-651.

MUFSON, L., Moreau, D., Weissman, M.M., Wickramaratne, P., Martin, J. \& Samoilov, A. (1994). Modification of interpersonal psycotherapy with depressed adolescents (ITP-A): phase I and II studies. Journal of American Academy of Child and Adolescent Psychiatry, 33 (5): 695-705.

MUFSON, L. \& Fairbanks, J. (1996). Interpersonal psychotherapy for depressed adolescents: a one-year naturalistic follow-up study. Journal of American Academy of Child andAdolescentPsychiatry, 35: 1145-1155.

MUFSON, L.; Weisman, M.M.; Moreau, D. \& Garfinkel, R. (1999). Efficacy of interpersonal psychotherapy for depressed adolescents.
Archives of General Psychiatry 56 (6): 573-579.

RACHMAN, A.W. \& Raubolt, R.R. (1984). The pioneers of adolescents group psychotherapy. International Journal of Group Psychotherapy 34: 387-413.

ROSSELO, J. \& Bernal, G. (1999). The efficacy of cognitive-behavioral and interpersonal treatments for depression in Puerto Rican adolescents. Journal of Consulting Clinical Psychology, 67:734-745.

SADLER, L.S. (1991). Depression in adolescents. Context, manifestations, and clinical management. Nursing Clinics of North America, 26: 559-572.

SCHESTATSKY, S. \& Fleck, M. (1999). Psicoterapia das depressões. Revista Brasileira de Psiquiatria, 21 (supl.): 41-47.

SCIVOLETTO, S.; Nicastri, S. \& Zilberman, M.L. (1994). Transtorno depressivo na adolescência: diagnóstico e tratamento. Revista Brasileira de Medicina, 51 (9): 1211-1228.

SHAFII, M. \& Shafii, S.L. (1992). Dynamic Psychotherapy of Depression. In M. Shafii \& S. L. Shafii (eds.) Clinical Guide to Depression in Children and Adolescents. Washington: American Psychiatric Press. pp. 157-175.

SON, S.E. \& Kirchner, J.T. (2000). Depression in children and adolescents. American Family Physician, 62: 2297-2308.

TASK Force on Promotion and Dissemination of Psychological Procedures. (1995). Training in and dissemination of empirically-validated psychological treatments: Report and recommendations. The Clinical Psychologist, 8: 3-24.

WEISSMAN, M.M. (1997). Interpersonal psychotherapy: current status. Keio Journal of Medicine, 46 (3): 105-110.

YALOM, I.D. (1975). The Theory and Practice of Group Psychotherapy. $2^{\text {nd }}$ ed. New York: Basic Books.

Recebido para publicação em 27 de setembro de 2002 e aceito em 12 de junho de 2003. 